\title{
Operation of Alcator C-Mod with high-Z plasma facing components and implications ${ }^{a)}$
}

\author{
B. Lipschultz, ${ }^{\text {b) }}$ Y. Lin, M. L. Reinke, A. Hubbard, I. H. Hutchinson, J. Irby, B. LaBombard, \\ E. S. Marmar, K. Marr, J. L. Terry, S. M. Wolfe, and the Alcator C-Mod group \\ M.I.T. Plasma Science and Fusion Center, 175 Albany Street, Cambridge, Massachusetts 02139 \\ D. Whyte \\ University of Wisconsin-Madison, 1500 Engineering Drive, Madison, Wisconsin 53706
}

(Received 29 October 2005; accepted 12 December 2005; published online 15 May 2006)

\begin{abstract}
Studies of potential plasma facing component (PFC) materials for a magnetic fusion reactor generally conclude that tungsten is the best choice due to its low tritium (T) retention, capability to handle high heat fluxes with low erosion, and robustness to nuclear damage and activation. ITER [F. Perkins et al., Nucl. Fusion 39, 2137 (1999)] may operate with all tungsten PFCs to provide the necessary operational experience for a reactor. Alcator C-Mod [I. Hutchinson et al., Phys. Plasmas 1, 1511 (1994)] operates with molybdenum (Mo) high-Z PFCs, which have very similar properties to tungsten. The experiments described herein have provided a unique comparison of operation with or without in situ boron coatings applied to the molybdenum PFCs; the latter are likely most relevant to ITER and beyond. ICRF-heated $H$-modes were readily achieved without boron coatings although the resultant enhancement in energy confinement was typically small $\left(H_{\mathrm{ITER}, 89} \sim 1\right)$. Molybdenum concentrations, $n_{\mathrm{Mo}} / n_{e}$, rise rapidly after the $H$-mode transition up to $0.1 \%$, cooling the plasma by line radiation, reducing energy confinement, and/or causing a back $H / L$ transition. Surprisingly, the primarily molybdenum PFC surfaces retain $3.5-5.0 \times 10^{20}$ of injected $\mathrm{D}_{2}$ molecules per discharge, corresponding to $50 \%$ of the injected gas. Plasma current disruptions, both randomly occurring over the course of a day, or planned, reduce the retained D long term. After applying boron coatings, $n_{\mathrm{Mo}} / n_{e}$ was reduced by a factor of $10-20$ with $H_{\mathrm{ITER}, 89}$ approaching 2 . A world-record volume-average plasma pressure of 1.8 atm at $5.4 \mathrm{~T}$ was achieved at the ITER normalized $\beta$. The effects of each boronization are found to be limited in time, correlated to time-integrated input energy. Intra- and inter-discharge boronization techniques have been developed with the latter being the most successful. This initial study indicates that a low- $Z$ coating over at least a fraction of the Mo PFCs in C-Mod is needed to reduce core molybdenum levels and achieve the best energy confinement. This, together with the larger than expected D retention, raises concerns for the performance of uncoated tungsten surfaces in ITER and beyond. () 2006 American Institute of Physics. [DOI: 10.1063/1.2180767]
\end{abstract}

\section{INTRODUCTION}

As we confront the task of building the ITER (Ref. 1) and the power-producing reactors that will follow, it is apparent that the proper selection of plasma facing components (PFCs) will strongly affect the performance of such devices. Most fusion reactor studies prefer tungsten over carbon due to its advantages with respect to neutron damage (swelling, void production, degradation of thermal and mechanical properties) as well as erosion. ${ }^{2-7}$ Another motivation for using tungsten is the level of tritium retention in tungsten is predicted to be lower by orders of magnitude ${ }^{8}$ than with carbon PFCs. However, relative to carbon PFCs, there is less operational experience with high- $Z$ materials. Alcator C-Mod, along with several limiter tokamaks [Frascati Tokamak Upgrade (FTU) and Tokamak Research Institute for Applied Mechanics (TRIAM)], have molybdenum PFCs. ${ }^{9-11}$ Molybdenum is similar to tungsten in that it has a high tem-

\footnotetext{
a) Paper FI1 3, Bull. Am. Phys. Soc. 50, 103 (2005).

${ }^{b)}$ Invited speaker.
}

perature limit and low tritium retention. ${ }^{12}$ Other tokamak programs have been experimenting with tungsten PFCs, ${ }^{13}$ most notably ASDEX-Upgrade where the carbon PFC tiles are gradually being replaced with tungsten-coated carbon tiles. ${ }^{14}$ If the experimental results from all such tokamaks lead to a positive assessment of high-Z PFCs, then ITER would provide the ultimate test of tungsten as close to reactor conditions as possible.

The choices of PFC materials for ITER are less limited than for a reactor because of lower neutron fluence levels. Carbon is placed in the region of the divertor strike points because it does not melt (a positive attribute) but is limited to that region to minimize the potential for tritium retention. ${ }^{15}$ Beryllium is used over much of the surfaces outside the divertor (main chamber) and tungsten is used for regions of the divertor outside the highest power fluxes to minimize concerns of damage due to disruptions and edge localized modes (ELMs).

Tokamaks are rarely operated using as-installed, bare PFCs. Instead, wall-coating methods are employed, involv- 
ing the deposition of thin $(\sim 100 \mathrm{~nm})$, low-Z layers. These layers are found to enhance plasma performance (energy confinement) through impurity suppression, lower radiative losses and changes in recycling. The principal method used is termed "boronization" (see Ref. 16, and references therein). Boronization has been shown to effectively getter oxygen, thereby suppressing it in the plasma, which reduces core plasma radiation and sputtering of PFC surfaces. Oxygen $(Z=8)$ in the plasma has been replaced with lower- $Z$ boron $(Z=5)$, which, due to its lower ionization potentials, radiates less inside the separatrix. However, the boron layer is quickly eroded by subsequent discharges in some areas and does not significantly suppress carbon in the scrape-off layer (SOL) or core plasmas for more than tens of discharges. ${ }^{16}$ An alternative wall coating, principally used in the Joint European Torus (JET), is beryllium, applied using evaporation techniques, again used with similar results. ${ }^{17,18}$

While the above conditioning techniques can in principle be applied to ITER or a reactor prior to operations, routine application of such surface coatings would not seem practical for high energy density, long pulse environments. This observation raises the concern that the confinement scalings developed to predict ITER and reactor performance may not be appropriate as they are based on data primarily from carbon-PFC tokamaks with low-Z coatings. Is energy confinement (and thus achievement of the required Q) for fully high-Z uncoated PFC operation in ITER or a reactor being properly predicted? It is clear that at some point (e.g., ITER) before construction of a demonstration fusion reactor (DEMO) the fusion community must gain confidence and experience in all high-Z PFC operation without low-Z wall coatings.

The Alcator C-Mod tokamak is well suited for comparing boronized to un-boronized high-Z PFC surfaces. Largescale removal of boron films from metal surfaces is relatively straightforward compared to carbon PFCs where abrasion/ machining or chemical treatment are likely needed. Parallel heat loads $\left(q_{\|} \sim 0.5 \mathrm{GW} / \mathrm{m}^{2}\right)$, as well as divertor density and temperature, are comparable to that predicted for ITER. The opacity of the SOL to neutrals (impurities and D) is similar to that predicted of ITER. ${ }^{19}$ Lastly, the radiative cooling rate for molybdenum at C-Mod core temperatures is the same magnitude as tungsten in the higher plasma temperature ITER core.

Another concern also motivated C-Mod experiments comparing high-Z PFC operation with and without boron coatings. C-Mod, like carbon PFC tokamaks, has made it standard practice to boronize the interior surfaces of the vessel (since 1996). During the period 2000-2004 the rf power required to achieve $170 \mathrm{~kJ}$ of stored thermal energy (red circles, Fig. 1) almost doubled; energy confinement appeared to have degraded. Also shown in Fig. 1 is the thickness of boron layers (blue squares), the estimate of which is made based on the amount of diborane used for each boronization and a surface area of $10 \mathrm{~m}^{2}$. Also shown is the point in time when boron-nitride (BN) tiles were installed on the antenna protection limiters. Was the degradation of achievable stored energy due to the fact that there were thick boron layers? BN tiles? Inefficiencies in ICRF heating? Other changes in the

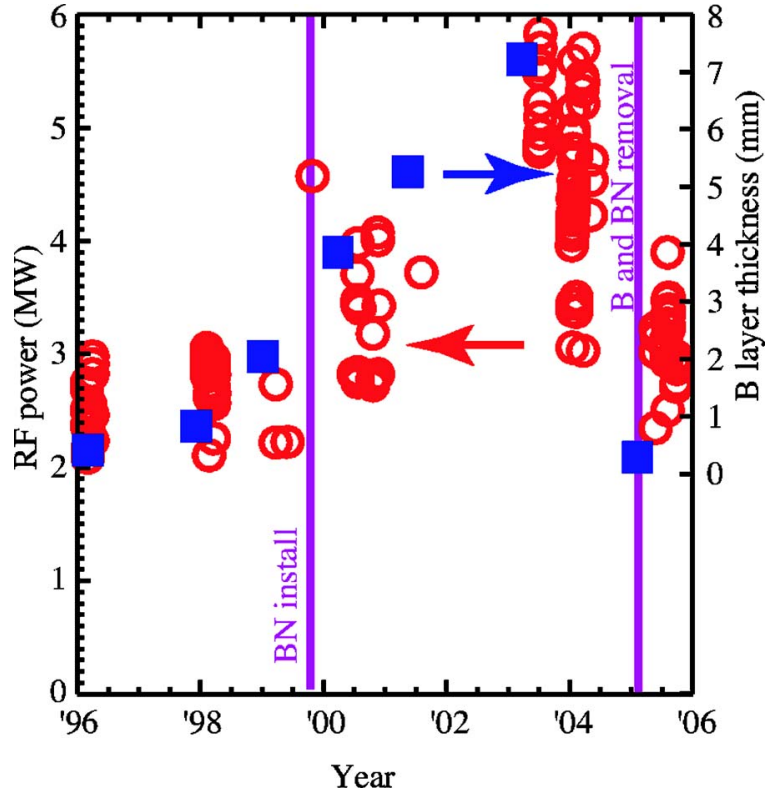

FIG. 1. (Color online) The amount of ICRF power required to obtain $160 \mathrm{~kJ} \leqslant W_{\mathrm{MHD}} \leqslant 170 \mathrm{~kJ}$ vs campaign period. The estimated boron layer thickness and date when the $\mathrm{BN}$ antenna protection tiles were added and subsequently removed are shown.

machine? Diagnostic calibrations? A number of these issues could be addressed only by removing B from the machine to recover pre-boronization conditions. This step was taken prior to the $2005 \mathrm{C}$-Mod experimental campaign and is described later in this paper.

An important variable potentially affecting performance is the control of hydrogen concentrations in the deuterium plasmas. Low $\mathrm{H} /(\mathrm{H}+\mathrm{D})$ levels are necessary for efficient ICRF heating as the rf waves are resonant with hydrogen minority ions in a D plasma. This is illustrated in Fig. 2 where the sawtooth reheat rate, a measure of the heating efficiency for fixed plasma density and fixed ICRF power

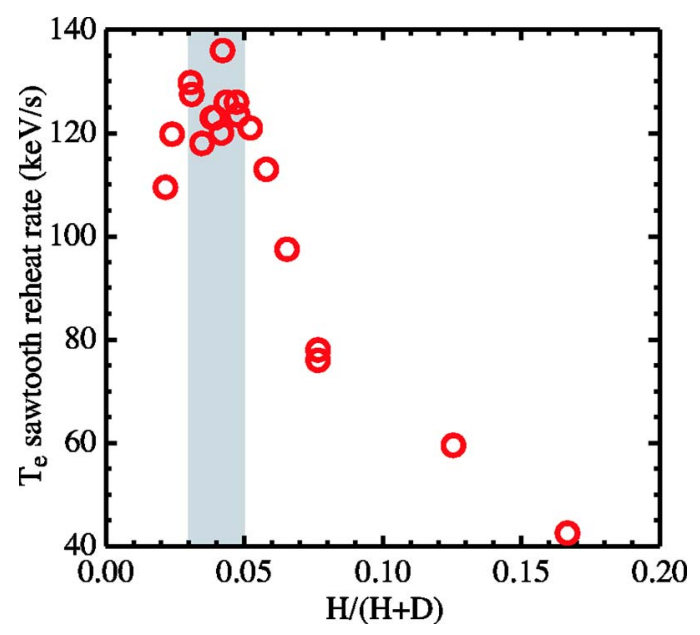

FIG. 2. (Color online) The sawtooth reheat rate for a set of discharges where the ICRF power launched and general plasma characteristics were held constant while the $\mathrm{H} /(\mathrm{H}+\mathrm{D})$ ratio was varied. The sawtooth reheat rate is then a measure of the local absorbed power and therefore the heating efficiency as well. The shaded region is the range in $\mathrm{H} /(\mathrm{H}+\mathrm{D})$ considered optimal for minority heating efficiency. 
launched, decreases with increasing $\mathrm{H} /(\mathrm{H}+\mathrm{D})$. The $\mathrm{H} /(\mathrm{H}$ $+\mathrm{D})$ ratio is determined through the ratio of the hydrogen and deuterium Balmer ${ }_{\alpha}\left(\mathrm{H}_{\alpha} / \mathrm{D}_{\alpha}\right)$ edge recycling line intensities as measured along one chord through the plasma (a view radially inward into the torus slightly below the outer midplane). Such a measurement is obviously not a measure of the core plasma $\mathrm{H} /(\mathrm{H}+\mathrm{D})$. Nevertheless, it provides a gross but effective indicator of the global $\mathrm{H} /(\mathrm{H}+\mathrm{D})$ ratios as witnessed by the good organization of ICRF heating efficiency versus the edge $\mathrm{H} / \mathrm{H}+\mathrm{D}$ ratio.

Hydrogen remains in the vessel after a vacuum break due to water from the air being adsorbed onto, and absorbed into, surfaces. During the 2000-2004 period the first boronization of each campaign was performed when $\mathrm{H} /(\mathrm{H}+\mathrm{D})$ had dropped to $\sim 10 \%$. The boronization application was found to lower the $\mathrm{H} /(\mathrm{H}+\mathrm{D})$ level to $\sim 5 \%$, the level needed to achieve efficient ICRF heating. However, $\mathrm{H} /(\mathrm{H}+\mathrm{D})$ would slowly rise after each boronization. The suspicion is that each newly deposited boron layer initially buries the $\mathrm{H}$ under a deuterated-boron layer. But, over time or with surface heating, the $\mathrm{H}$ diffuses to the new boron surface where it can recombine into volatile $\mathrm{H}_{2}$ or $\mathrm{HD}$ and be released from the surface. Thus, removing the boron layers was thought to be a good way of regaining control of this important parameter. Indeed, the results from the current 2005 experimental campaign ${ }^{20}$ confirm that the high $\mathrm{H} /(\mathrm{H}+\mathrm{D})$ levels correlate with poor ICRF heating efficiencies during the 2000-2004 campaign. Removal of the thick boron layers and/or installed BN tiles, detailed in this paper, allow the recovery of pre2000 performance (see Fig. 1, 2005). We conclude that the presence of thick boron layers or bulk BN somehow affects the long-term trapping and removal of the $\mathrm{H}$ in the wall.

\section{EXPERIMENT DESCRIPTION}

Basic characteristics of the Alcator C-Mod tokamak experiment, ICRF heating, and diagnostics are described elsewhere. ${ }^{21-23}$ The data used in this study were acquired with $5.3 \mathrm{~T}$ toroidal magnetic field at the plasma center and plasma currents generally close to $1.0 \mathrm{MA}$. All discharges were diverted with a lower single null magnetic topology. Shown in Fig. 3 are the standard tokamak plasma-interaction surfaces comprised of the order of 7000 molybdenum tiles. The inner wall generally experiences high heat loads only during plasma startup during the first $150 \mathrm{~ms}$ of each discharge. In addition there are two partial poloidal limiters (one split into two pieces) at the outside edge of the plasma as well as tiles directly attached to the outside edge of the antennas ("antenna protection tiles").

Spectroscopic measurements are utilized to monitor the impurity radiation as well as infer the impurity densities. Standard bolometer arrays are used for measurement of the core plasma radiation. ${ }^{24,25}$ In addition there are " $2 \pi$ " bolometer detectors in an outer port that accept light from $2 \pi$ steradians for global radiation levels. ${ }^{25}$

Vacuum ultraviolet (VUV) measurements were made with a $2.2 \mathrm{~m}$, Rowland circle, grazing incidence spectrometer having a spectral resolution of $0.06 \mathrm{~nm}$ at $10 \mathrm{~nm}$. The principal emission lines used to monitor core molybdenum

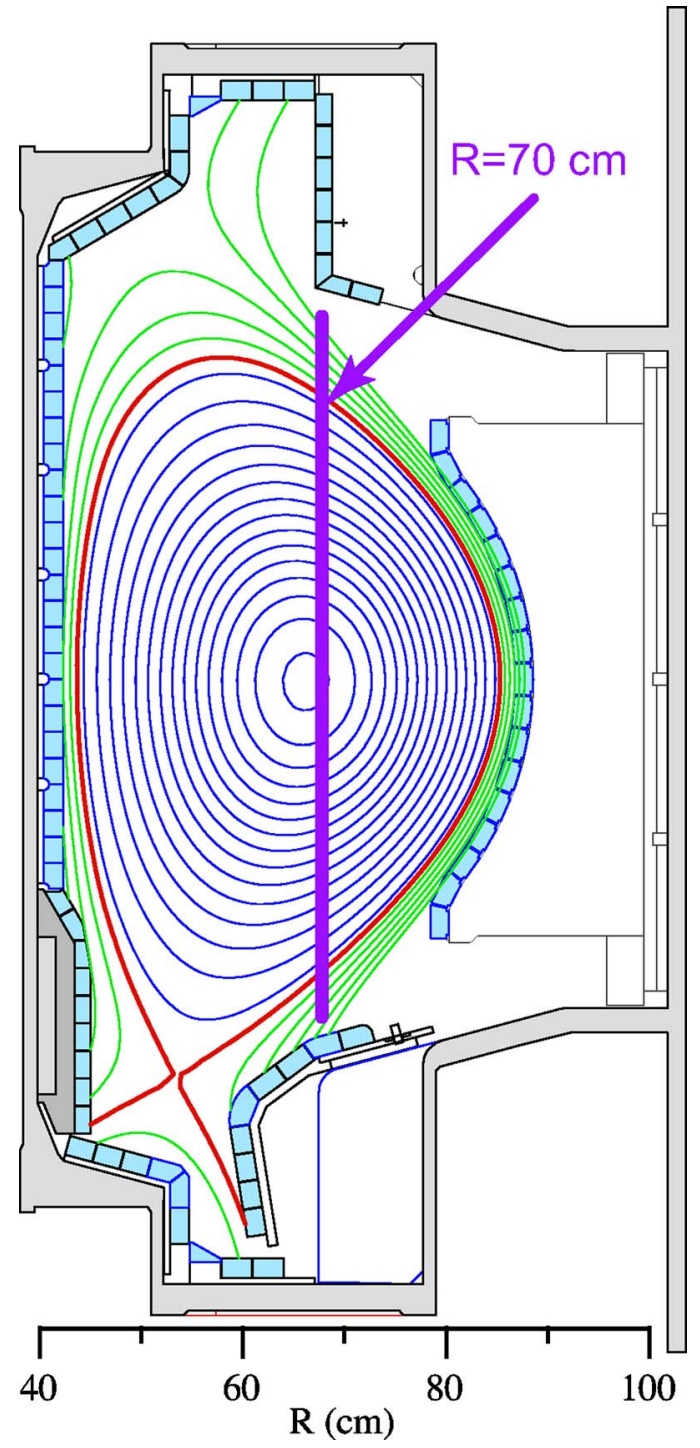

FIG. 3. (Color online) Cross section of the Alcator C-Mod vessel. The molybdenum tiles are shown in blue. The inner wall is located at $R$ $=44 \mathrm{~cm}$. The largest major radius edge of the inner divertor is at $46.8 \mathrm{~cm}$. The smallest major radius edge of the outer divertor, or outer divertor nose is at $61.2 \mathrm{~cm}$. The largest major radius location on the outer divertor is at $75.6 \mathrm{~cm}$. The smallest/largest major radii of the outer limiter tiles are 81.8 and $90.5 \mathrm{~cm}$, respectively. Finally, the outer vessel wall is at $103.7 \mathrm{~cm}$. The vertical line is at $R=70 \mathrm{~cm}$.

and iron radiation were Mo XXXI $(11.599 \mathrm{~nm})$ and $\mathrm{Fe}$ XXIII $(13.285 \mathrm{~nm})$. The plasma densities for molybdenum and iron were inferred using the measured line brightnesses, Thomson scattering electron density and temperature profiles, the MIST impurity transport code, ${ }^{26}$ transport coefficients, ${ }^{27}$ and cooling curves. ${ }^{28-30}$

Visible measurements were made with a $0.25 \mathrm{~m}$ focal length instrument having a spectral resolution of $\sim 0.25 \mathrm{~nm}$. Its primary purpose in this study was for monitoring of fully striped boron and fluorine densities near the top of the $\mathrm{H}$-mode pedestal (6 $\mathrm{mm}$ inside the separatrix) by simultaneous measurement of the B V $(494.4 \mathrm{~nm})$ and F IX $(474 \mathrm{~nm})$ lines. The lines were excited via charge-exchange from deuterium in the $n=2$ level at the inner edge of the plasma by injection of room temperature $\mathrm{D}_{2}$ neutrals from a 
$1 \mathrm{~mm}$ diameter capillary tube located at the inner midplane $1-1.5 \mathrm{~cm}$ from the separatrix. Light from a set of six toroidal viewing chords perpendicular to the neutral injection is transmitted to the spectrometer.

\section{BORONIZATION HISTORY}

The C-Mod boronization procedure employs an electron cyclotron discharge cleaning (ECDC) plasma discharge with a helium-diborane gas mixture $\left(10 \% \mathrm{~B}_{2} \mathrm{D}_{6}, 90 \% \mathrm{He}\right)$. The plasma is created at the major radius corresponding to the electron cyclotron resonant magnetic field $(f=2.45 \mathrm{GHz}, B$ $=0.088 \mathrm{~T})$ which is varied to sweep the resonance across the chamber. A plasma of fairly constant density and temperature extends outward in major radius from the cylindrical resonance location, thus having some effect on all radii outside the resonance. ${ }^{31}$ The plasma density drops off quickly ( $\sim 3 \mathrm{~mm}$ e-folding distance) towards smaller major radius. Toroidal uniformity is maintained by releasing the diborane gas into the vessel from a toroidal pipe located at the top of the vessel with holes spaced $\sim 1 \mathrm{~cm}$ toroidally. The boronization period is $\sim 12 \mathrm{~h}$ at a typical deposition rate of $\sim 10 \mathrm{~nm} / \mathrm{h}$ assuming a $10 \mathrm{~m}^{2}$ deposition area. The microwave power is modulated with a $50 \%$ duty cycle to allow the diborane gas to circulate between ECDC periods. The same ECDC plasma, however with pure $\mathrm{D}_{2}$ or $\mathrm{He}$ gas and $100 \%$ duty cycle, is used to clean the walls after a vacuum break or before a set of experiments. The boronization process is repeated when plasma radiation becomes problematic and degrades energy confinement.

There is direct evidence of boron erosion. Postboronization Mo I $(386.4 \mathrm{~nm})$ spectroscopic measurements show that the outer divertor molybdenum influx is initially absent, but then, as the boron layer erodes over tens of discharges, the Mo influx recovers to pre-boronization levels. ${ }^{32}$ More importantly, we suspect that the Mo erosion is occurring on surfaces outboard of the divertor where rf sheath rectification leads to large potentials and sputtering of surface material. ${ }^{32}$

While spectroscopy indicates rapid erosion of boron, the erosion seems isolated to a small fraction of the wall. The majority of PFC surfaces in C-Mod experience little or no net erosion, or even net deposition. Sample tiles from a poloidal circumference of the vessel were removed for surface analysis prior to the 2005 experimental campaign. Boron layers in the range of $6 \mu \mathrm{m}$ thick have been measured on all tiles except for the outer and inner divertor tiles. The average boron layer thickness on inner divertor tiles, which had been installed for only 3 years of operation, was smaller $(\sim 2-3 \mu \mathrm{m})$. The outer divertor surfaces had the lowest boron surface content (equivalent layer $<1 \mu \mathrm{m}$ ) and the highest Mo surface concentrations $(10 \%-50 \%)$, presumably due to net erosion of the boron layers and well correlated with spectroscopic measurements discussed above. These measured regions of "plasma-cleaned" surfaces extend from the bottom of the outer vertical target to $R \sim 0.67 \mathrm{~m}$ on the outer horizontal shelf of the divertor (see Fig. 3). The largest molybdenum fraction, corresponding to the largest boron erosion, is near the usual strike point. The pattern of erosion described is similar to the results of a previous study. ${ }^{33}$

A number of steps were taken to remove as much boron from the C-Mod vessel as possible before the 2005 campaign, which is the primary focus of this paper. All BN antenna protection tiles were removed and replaced with Mo tiles. All limiter tiles and upper divertor tiles were removed and ultrasonically cleaned. There were a number of other tiles and surfaces that were either difficult (e.g., inner divertor) or impossible (e.g., stainless steel walls) to remove for cleaning. Those surfaces were carefully wiped with dustand lint-free cloths and deionized water to remove as much boron as possible. The amount of boron remaining on tiles was very low as determined from nuclear reaction analysis measurements; $10 \%-15 \% \mathrm{~B} /$ Mo distributed through the first micron, either trapped in the topography of the surface or in grain boundaries. The wiped tiles had slightly more boron coverage of $20 \%-30 \%$. In any case the cleaned tile surfaces had predominately Mo at the surface, a stark difference to the typical post-boronization surface with thick boron layers and $\sim 1 \%$ Mo and other trace impurities like oxygen.

\section{INITIAL OPERATION WITHOUT BORONIZATION}

The first step in preparing C-Mod for physics operation after a vacuum vent is to reduce the hydrogen concentration in the plasma. During a vacuum break water $\left(\mathrm{H}_{2} \mathrm{O}\right)$ is adsorbed onto the tile surfaces and $\mathrm{H}$ is absorbed into the tiles. After pump down water dominates the RGA spectrum. The vessel is baked at $120^{\circ} \mathrm{C}$ for several days, limited in temperature by the magnetic equilibrium coils. Wall cleaning, utilizing ECDC and $\mathrm{D}_{2}$ gas, is then started simultaneous with vessel baking. Large amounts of $\mathrm{H}_{2}\left(\sim 5 \times 10^{22} \mathrm{H}_{2}\right.$ molecules) are removed quickly during the first few days of ECDC wall conditioning. The $\mathrm{H}_{2}$ removal rate decreases with an e-folding time of $\sim 1$ day. Following this period almost all $\mathrm{H}$ removal from tiles appears to be in the form of $\mathrm{HD}$, implying isotope exchange in the near surface is important; impacting $\mathrm{D}^{+}$ions and $\mathrm{D}$ atoms combine with $\mathrm{H}$ atoms in the surface to form volatile molecules.

Once this initial wall conditioning is done, tokamak discharges are started, as this is more efficient for $\mathrm{H}_{2}$ removal. The $\mathrm{H} /(\mathrm{H}+\mathrm{D})$ level in plasmas as a function of discharge number after a vent is shown in Fig. 4 for several experimental campaigns [The $\mathrm{H} /(\mathrm{H}+\mathrm{D})$ measurement was not available pre-1999]. We see that the initial removal rate of absorbed $\mathrm{H}$ is similar between bare molybdenum and heavily boron-coated surfaces, even with quite thick boron layers (2004). However, when the B layers were the thickest (2004), H/(H+D) kept rising above the optimal range for ICRF heating efficiency, dropping again as further boronizations were applied. This correlated with plasmas having reduced energy confinement properties as described in Sec. I.

Early in the campaign (prior to the first boronization), as the rf power reaches significant power levels, the $\mathrm{H} /(\mathrm{H}$ $+\mathrm{D})$ level tends to rise during each discharge, as well as from discharge to discharge through the run day. Our interpretation of this behavior is that as more energy is delivered to PFC surfaces, their maximum surface temperature will 


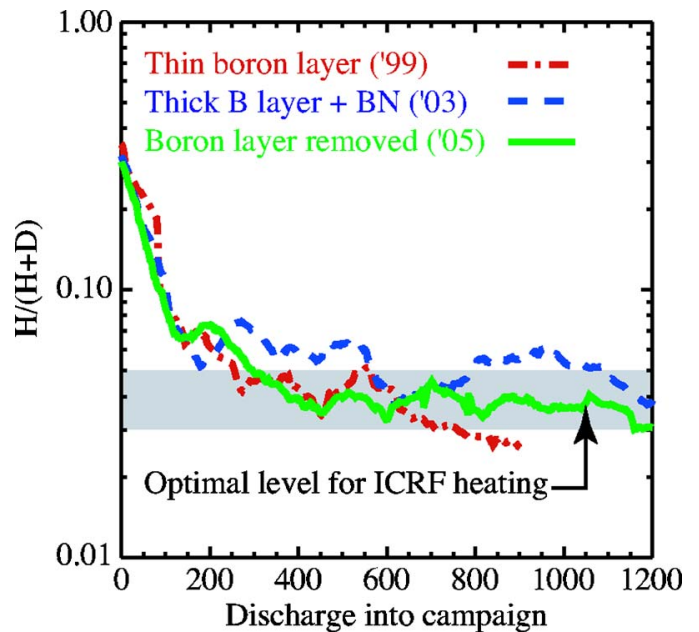

FIG. 4. (Color online) The history of the hydrogen fraction in the plasma, $\mathrm{H} /(\mathrm{H}+\mathrm{D})$, as a function of discharge number following a vacuum vent. The grey band spanning $3 \%-5 \%$ is the ideal level for efficient H-minority ICRF heating. Three cases are shown: 1999, before BN tiles were installed and the boron layer thickness on molybdenum tiles was thin (1-2 $\mu \mathrm{m}) ; 2003-2004$, when the boron layer thickness was largest ( $\sim 6 \mu \mathrm{m}$ microns); and 2005, when the boron layers were removed.

increase. $\mathrm{H}$ diffusivity and recombination rates in the surface increase exponentially with temperature ${ }^{34,35}$ forcing more $\mathrm{H}$ to the surface, leading to faster $\mathrm{H}$ removal, and increasing the $\mathrm{H}$ level in the plasma [and $\mathrm{H} /(\mathrm{H}+\mathrm{D})]$. In a similar vein, disruptive discharge cleaning experiments, where planned disruptions are aimed at heating specific surfaces, was very efficient in removing $\mathrm{H}$ and $\mathrm{D}$, and, as with $\mathrm{ECDC}$, in the form of HD.

When ICRF input powers reached $2 \mathrm{MW}$ and $\mathrm{H} /(\mathrm{H}$ $+\mathrm{D})$ levels dropped to $\sim 5 \%$ and did not significantly rise during the rf pulse, the 2005 physics campaign to investigate plasma energy confinement and general operation with uncoated molybdenum surfaces commenced. $H$-modes were readily achieved, although at least initially, the threshold powers required were high and the $H$-modes brief, with quick $H-L$ back-transitions caused by too much radiation. Later in the pre-boronization operational period the $H$-mode power threshold was lower, with ELM-free Ohmic $H$-modes $\left(H_{\text {ITER }, 89} \sim 1.5\right)$ (Ref. 36) occurring even at toroidal fields of $5.3 \mathrm{~T}$, indicating very clean target plasmas. Rising radiation led to $H$-Lback-transitions even for such Ohmic $H$-modes. Fairly steady state rf heated $H$-modes were eventually achieved. These generally had $L$-mode-like energy confinement $\left(H_{\text {ITER,89 }} \sim 1\right)$ and high radiation, although some reached $H_{\text {ITER }, 89} \sim 1.2-1.3$.

Efforts were made to minimize the radiation and thus improve energy confinement. Varying the magnetic equilibrium to increase the gaps between the plasma and various structures outside of the divertor did not appear to have any effect. $\mathrm{D}_{2}$ gas, injected during the $H$-mode to raise the density and cool the SOL, thereby minimizing impurity sputtering of PFCs, did lower the core molybdenum density. However the total radiated power only decreased slightly $\left(P_{\mathrm{RAD}}\right.$ $\left.\propto n^{2}\right)$ with a negligible improvement in energy confinement. Lastly, $\mathrm{N}_{2}$ injection into the divertor plasma led to divertor detachment and divertor molybdenum source rate dropping below the detection limit. The core molybdenum level, however, was unaffected, indicating that the vertical section of the outer divertor plate near the strike-point was not the dominant source of molybdenum reaching the core plasma. This result is consistent with a previous study ${ }^{32}$ showing that the probability of molybdenum impurities eroded from the outer vertical plate region reaching the core plasma was up to a factor of 100 times lower than for impurities trying to penetrate the SOL at the outer midplane. In summary, none of these efforts was successful in significantly improving the pre-boronization plasma performance.

Just prior to boronization of molybdenum PFC surfaces in the vessel, several alternative methods of conditioning the walls were tried. Li pellets were injected into discharges in an unsuccessful attempt to reproduce the improvement in energy confinement achieved in TFTR. ${ }^{37}$ The final attempt at conditioning the uncoated walls was to add $\mathrm{B}$ dust to the plasma - the hope being that it would be deposited where the plasma contacts. A reservoir of $100 \mu \mathrm{m}$ diameter boron "dust" was shaken during the discharge to release $\sim 5$ $\times 10^{20}$ boron atoms per discharge of which we estimate at roughly $10 \%$ makes it into the core plasma. There was no discernible enhancement in confinement, reduction of molybdenum source rates, or core radiation. Either the boron is being eroded faster than it is deposited (no net deposition) or it is not being deposited at the molybdenum erosion sites affecting the core molybdenum levels. On the other hand, the boron levels in the plasma were increased greatly (factor $>10$ ) to levels of $n_{\mathrm{B}} / n_{e} \sim 1 \%$, similar to that found in postboronization (see Sec. IV). This indicates that boron by itself does not lead to an enhancement of confinement.

\section{POST-BORONIZATION PLASMA PERFORMANCE}

After fully characterizing operation with un-boronized PFC surfaces, the first ECDC boronization of the campaign was conducted. It was limited to covering the regions of the outer divertor and outwards in the major radius (see Fig. 3, $R=50-90 \mathrm{~cm}$ ). The energy confinement was immediately enhanced simultaneous with reduced molybdenum levels in the plasma. The performance improvement from this first boronization did not last long so a second boronization was performed. Molybdenum levels were reduced further and energy confinement again improved. The waveforms for three discharges illustrative of pre-boronization, post first boronization, and after the post second boronization, are shown in Fig. 5. The pre-boronization discharge started in the $H$-mode with a rapid increase in density and radiation. An equilibrium was then reached with marginally improved particle confinement, as evidenced by the very small increase to the final $H$-mode density, but poor energy confinement. There was a definite improvement after the first boronization, with increased stored energy and reduced radiation. Both the density and the radiation rose more slowly at the beginning of the $H$-mode. The stored energy, although initially high, dropped as the radiation continued to rise. Finally, after the second boronization, the combination of higher powers and lower radiation led to the best discharge performance. 


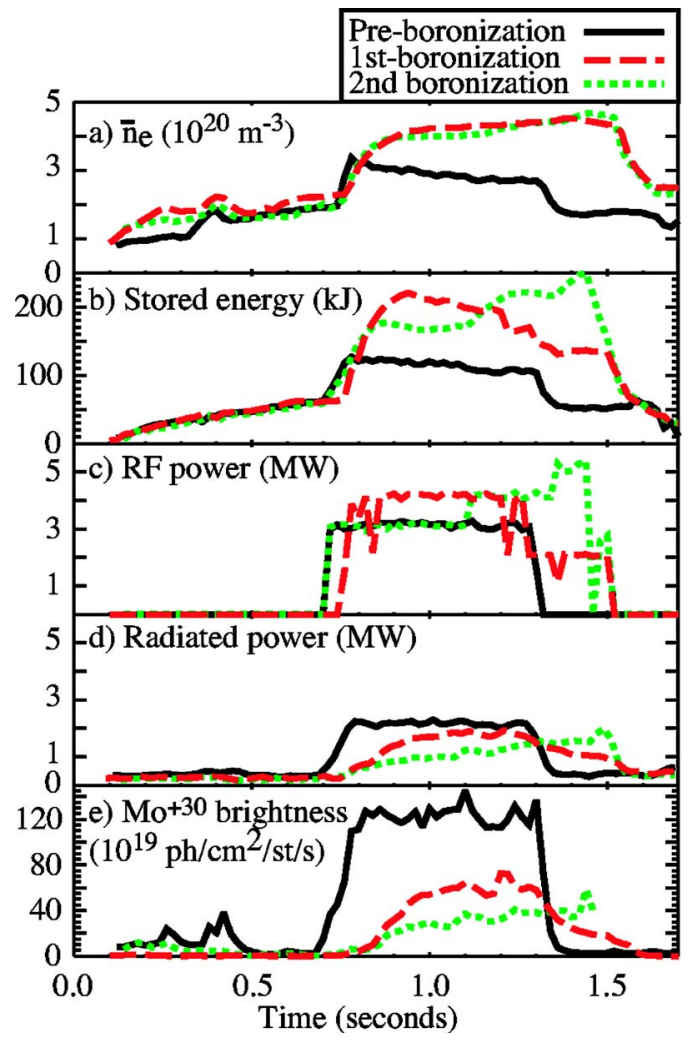

FIG. 5. (Color online) Examples of discharges from pre-boronization (black), post-first-boronization (red), and post-second-boronization (green). The traces for a number of parameters are given: (a) line-averaged density; (b) stored energy; (c) rf power (source); (d) radiated power from the plasma inside the separatrix (not the divertor); and (e) brightness of a Mo XXXI line $(11.599 \mathrm{~nm})$.

The discharge following the second boronization that is shown is significant from another point of view; the volumeaveraged plasma pressure is 1.8 atm, a new record for C-Mod and, we believe, for tokamak plasmas. The $\beta_{N}$ of this plasma is 1.7 , similar to the target $\beta_{N}$ for ITER, with the same toroidal field as C-Mod. The $q_{95}$ for the ITER design point is lower, and the corresponding volume-averaged pressure would be 2.8 atm.

The effect of radiation can be better understood by examining the relationship among the confinement $H$ factor,

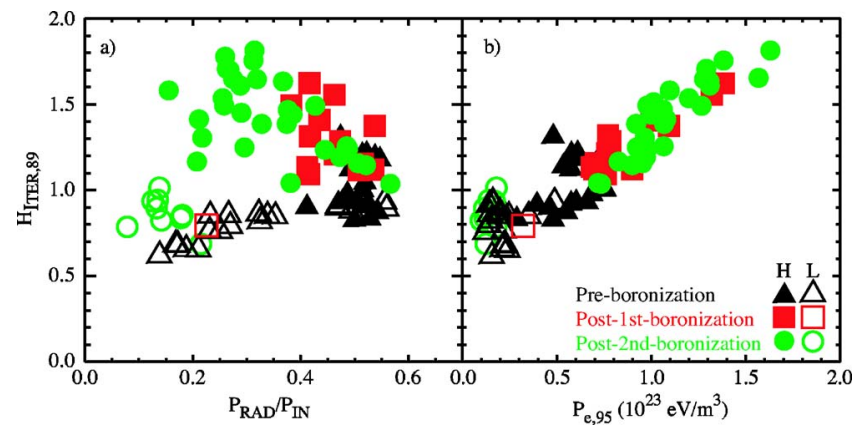

FIG. 6. (Color online) Comparison of confinement characteristics before (black), post-first-boronization (red), and post-second-boronization (green). $L$-mode points are unfilled, $H$-mode points are filled. (a) $H_{\text {ITER,89 }}$ vs the radiated power fraction; (b) $H_{\text {ITER,89 }}$ vs the pedestal electron pressure $\left(3 n_{e} T_{e} / 2\right)$.

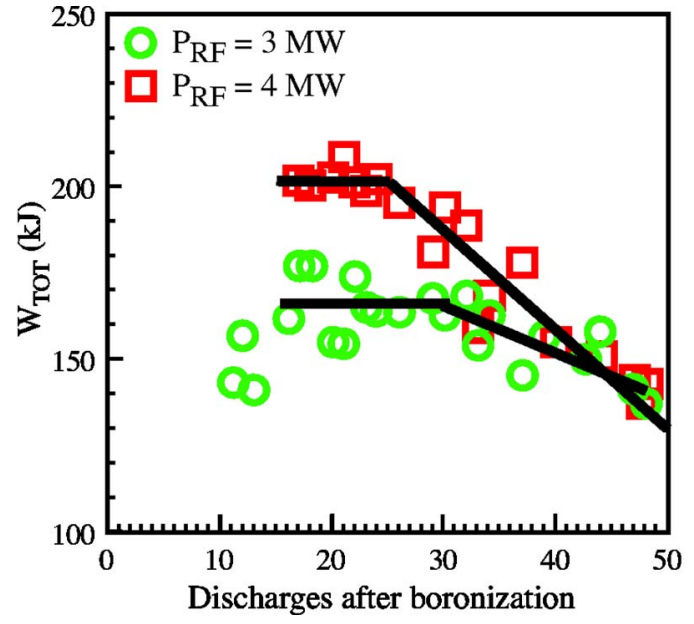

FIG. 7. (Color online) Plotted is the stored energy vs discharge sequence number after an overnight boronization for two ICRF power levels.

radiation, and the pedestal pressure. Figure 6(a) displays the characteristics of a set of time points from discharges with "steady state" conditions; radiation, $H_{\mathrm{ITER}, 89}$, core density, and temperature are approximately constant for at least $200 \mathrm{~ms}$ (3-5 energy confinement times). The highest $H_{\text {ITER, } 89}$ values are for radiated power fractions, $P_{\mathrm{RAD}} / P_{\mathrm{TOT}}$, of $\sim 0.25$ and then decrease as the radiated power fraction increases, a dependence similar to that found in a previous C-Mod study. ${ }^{38}$ The pre-boronization $H$-modes, having high radiated power fractions and low $H_{\text {ITER,89 }}$ values, fit well with the general trend in post-boronization data points. $L$-mode values of $H_{\text {ITER,89 }}$ below 1 are indicative of either poor assessment of rf power absorption (80\% rf power absorbed by the plasma was assumed for all discharges) or the $H_{\text {ITER,89 }}$ scaling not properly fitting C-Mod data for auxiliary heating.

The reason for degradation in energy confinement with large radiated power fractions is the degradation of the pedestal. Shown in Fig. 6(b) are pedestal pressures at the $\psi_{95}$ $(\psi=95 \%)$ flux surface from the same set of discharges as shown in Fig. 6(a). At high radiated power fractions $H_{\text {ITER,89 }}$ is low, corresponding to low pedestal pressures. Such a scaling is to be expected based on profile stiffness. ${ }^{38-41}$ The net power reaching the pedestal region, $P_{\mathrm{NET}} \sim P_{\mathrm{TOT}}-P_{\mathrm{RAD}, \mathrm{CORE}}$, also correlates well with pedestal pressure. The best energy confinement is for high $P_{\mathrm{NET}}$ and low $P_{\mathrm{RAD}, \mathrm{CORE}} / P_{\mathrm{TOT}}$ as expected from a simple heat conduction argument for a fixed pedestal width.

A directed effort was made to determine the optimal number of high-performance discharges that could be obtained between boronizations. After an overnight boronization, constant discharges were programmed with as similar characteristics as possible. Each discharge included an ICRF power waveform with several levels of rf power. The stored energy achieved at a given rf power decreased (see Fig. 7) roughly midway through the 50 discharge run day as the impurity levels increased. The start of the decrease in $W_{\mathrm{TOT}}$ corresponded to $\sim 50 \mathrm{MJ}$ of injected energy integrated across discharges. Review of the confinement trends postboronization in past run campaigns reveals a turnover in 


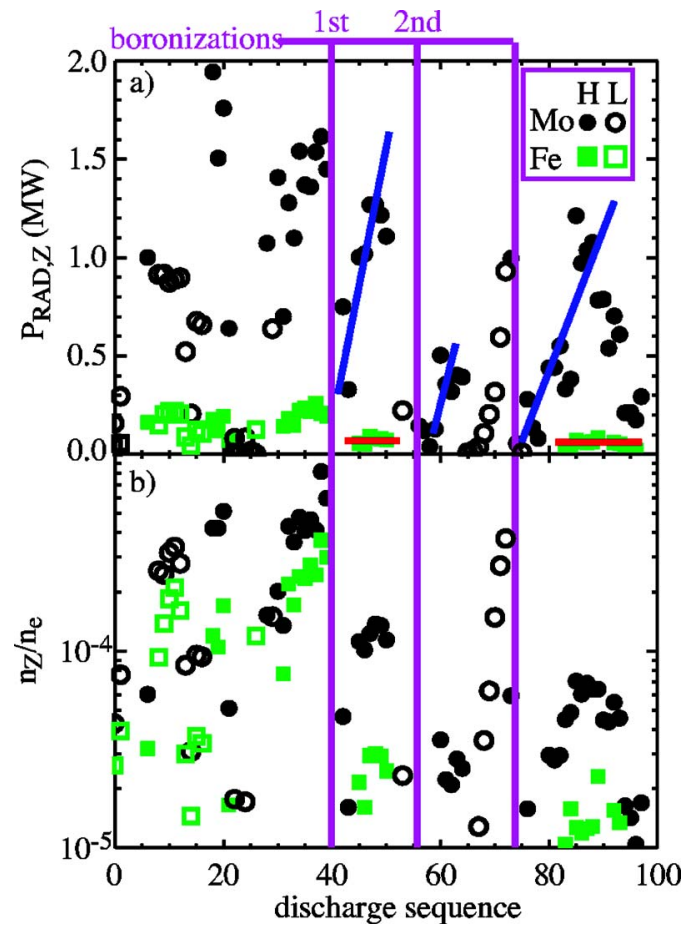

FIG. 8. (Color online) The effect of boronization on impurities vs discharge sequence. Only steady state $H$-mode discharges where the VUV instrument was tuned to the proper wavelength to cover iron and molybdenum are included. Boronizations are indicated as solid vertical lines. (a) Radiated power for molybdenum (circles) and iron (squares); (b) impurity fraction for molybdenum (circles) and iron (squares).

achievable stored energy at a similar level of integrated input energy. This result implies that the boron film net erosion rate at the primary molybdenum source location is proportional to the amount of power injected. The e-folding "time" in terms of stored energy or $H_{\text {ITER }, 89}$ is in the range of 30 discharges (Fig. 7) or $\sim 50 \mathrm{~s}$ discharge time. This boron erosion period is similar to that measured for boronized carbon PFC machines ${ }^{16}$ where, as the boron is eroded, core levels of carbon rose back to pre-boronization levels. However the similarity ends there. When boron is replaced by carbon in the plasma the effect on core radiation is small. The effect on radiation is much more dramatic on C-Mod when the boron layer wears off. Molybdenum radiation and $P_{\mathrm{RAD}} / P_{\mathrm{TOT}}$ both rise concomitant with a drop in $H_{\mathrm{ITER}, 89}$.

\section{IMPURITY CHARACTERIZATION}

The radiation from both molybdenum $(Z=42)$ and iron $(Z=26$, from the stainless steel vacuum vessel and support structures) was investigated in the course of this experimental campaign. Each boronization leads to a drop in both the molybdenum and iron radiation [Fig. 8(a)]. The ability to derive the iron radiation was not available for all discharges but, based on later discharges, it remains low. Note that following each of the three boronizations shown (vertical lines) the molybdenum radiation increases throughout the day as the boron coating wears off. This increase in radiation corresponds to the drop in stored energy $\left(W_{\mathrm{TOT}}\right)$ after boronization shown in Fig. 7. No such trend following boronizations is observed for the iron radiation.
The molybdenum and iron fractional densities show a similar trend to the radiation data [Fig. 8(b)]. However, since pre-boronization $H$-mode densities are particularly low (e.g., see Fig. 5), the changes in radiation observed in Fig. 8(a) translate to larger changes in impurity fractions. We infer from the different trends in molybdenum vs iron radiation and densities that these impurities primarily come, as expected, from different locations. The stainless steel (primarily iron) vacuum vessel surfaces appear to remain adequately coated during continued operation, consistent with their larger distance from the plasma and less direct plasma contact. Based on the thick boron coatings found on most C-Mod tiles after 2004, it is also true that the majority of the molybdenum PFC surfaces remain covered by boron. But it is also clear that the erosion of the boron coating is much more rapid at the specific molybdenum source locations affecting the core, however few or small area they are.

The rapid rise in core molybdenum levels following boronization is unlikely to be attributed to changing $\mathrm{H} /(\mathrm{H}$ $+\mathrm{D})$ levels, From before the first boronization onward through this campaign the $\mathrm{H} /(\mathrm{H}+\mathrm{D})$ stayed fairly constant in the $3 \%-4 \%$ range even for high rf powers. It therefore seems unlikely that during that period higher $\mathrm{H} /(\mathrm{H}+\mathrm{D})$ levels led to higher ICRF deposition in the SOL, higher molybdenum source rates and enhanced molybdenum levels in the core plasma.

We can make simple estimates of the molybdenum influx and surface areas being eroded that are required to explain the observed core molybdenum levels. Using an $n_{\mathrm{Mo}} / n_{e}$ level of $5 \times 10^{-5}$ shown in Fig. 8(b), a core electron density of $4 \times 10^{20} \mathrm{~m}^{-3}$ and an impurity confinement time of $0.1 \mathrm{~s},{ }^{27}$ the corresponding Mo influx is $2 \times 10^{17} / \mathrm{s}$. Furthermore, using typical sputtering rates, the probability of Mo penetration into the core $(\sim 0.1$ from the outer SOL, Ref. 32$)$ and ion fluxes to surfaces, we estimate that a surface area in the range from 100 to $1000 \mathrm{~cm}^{2}$ is sufficient to provide the needed molybdenum source. If the sheath voltage drop is determined by sheath rectification, then much higher voltages and sputtering rates are likely. Estimates of surface areas required assuming sheath rectification are thus even lower (e.g., $10 \mathrm{~cm}^{2}$ ) as estimated previously. ${ }^{32}$

A large fraction of the plasma radiation can be attributed to iron and molybdenum radiation. Figure 9 displays the total radiation contribution from iron and molybdenum vs the core plasma radiated power determined from the bolometer arrays. During the pre-boronization period molybdenum can account for most of the plasma radiation. Iron accounts for another $10 \%-15 \%$ of the total radiation.

The data of Fig. 9 show that the molybdenum and iron radiation levels, following the second boronization, are typically lower than before and account for $\leqslant 50 \%$ of the core radiation. The iron radiation is quite low at this stage $(\sim 5 \%)$. Boron is a likely candidate to supply the remaining radiation as its density rises with boronization. $L$-mode boron levels (see Sec. II) are low but measurable $(0.05 \%-0.07 \%)$ before boronization, consistent with the finite amounts of boron remaining on surfaces after cleaning. The $n_{\mathrm{B}} / n_{e}$ level increases over the first two boronizations of the order of $0.3 \%$ in the $L$-mode and $\sim 1 \%$ in $H$-modes. The fluorine 


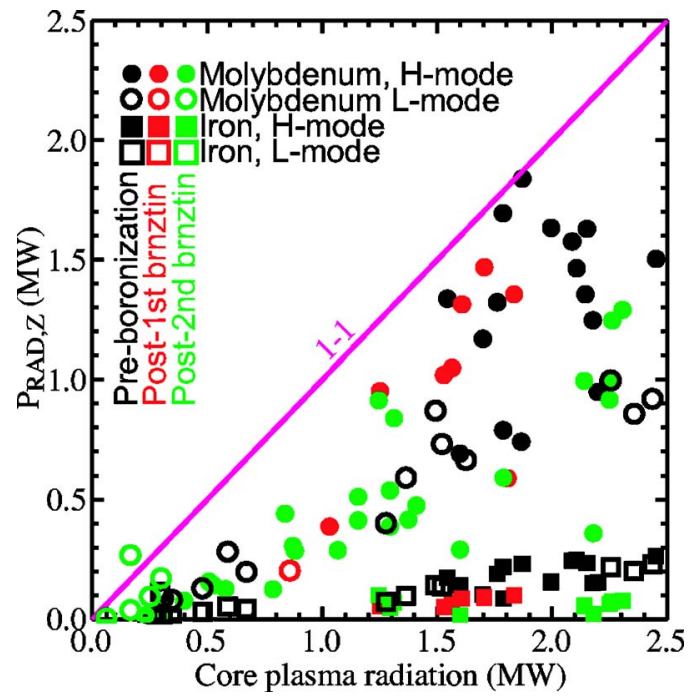

FIG. 9. (Color online) Power radiated by molybdenum (circles) and iron (squares) vs total radiated power. Same color coding as for Fig. 6. The molybdenum and iron radiation are generally lower after boronization (second).

level $\left(n_{\mathrm{F}} / n_{e} \sim 0.2 \%\right.$ in the $H$-mode), whose likely source is Teflon, is lower than for boron, but does not drop across a boronization as iron does. It would seem that it is not being eroded from surfaces. More likely it is in gaseous form. Using charge-state derived cooling rate curves ${ }^{42}$ we have estimated that fluorine radiation during the $H$-modes is in the range of $200-300 \mathrm{~kW}$ throughout the run period. Postboronization, the $H$-mode boron radiation is of the order of $100-200 \mathrm{~kW}$. These numbers are uncertain both because of uncertainties in the measurement and due to uncertainties in transport coefficients used to determine the charge state radial profiles in the plasma.

In summary, pre-boronization, molybdenum dominates the radiated power with significant contributions from iron and fluorine. Post-boronization, molybdenum is still a major radiator in the plasma, with fluorine and boron also playing a role. The only other potential contributor to radiation we have identified is titanium originating from the leading edge of a lower hybrid current drive launcher. Our best estimate is that $\mathrm{Ti}$ radiation does not exceed $150 \mathrm{~kW}$ for the discharges included in this study.

\section{RECYCLING CHARACTERISTICS}

We have also investigated the effect of boronization on plasma fueling, SOL characteristics and divertor characteristics. The initial studies of boronization (e.g., Refs. 43-45) showed that it led to a near-term decrease in the wall recycling coefficient; the wall pumped during the discharge. The results from C-Mod differ from those initial studies as can be seen in Fig. 10. The data shown are from a series of 1 MA, ICRF-heated discharges following an overnight boronization (followed by $4 \mathrm{~h}$ of ECDC in $\mathrm{He}$ and $2 \mathrm{~h}$ of ECDC in $\mathrm{D}_{2}$ ). Only the density prior to the $H$-mode period of the discharge [Fig. 10(d)] is controlled as the gas feed is turned off immediately after the $H$-mode transition. Changes in rf power, which occur discharge to discharge, did not affect the

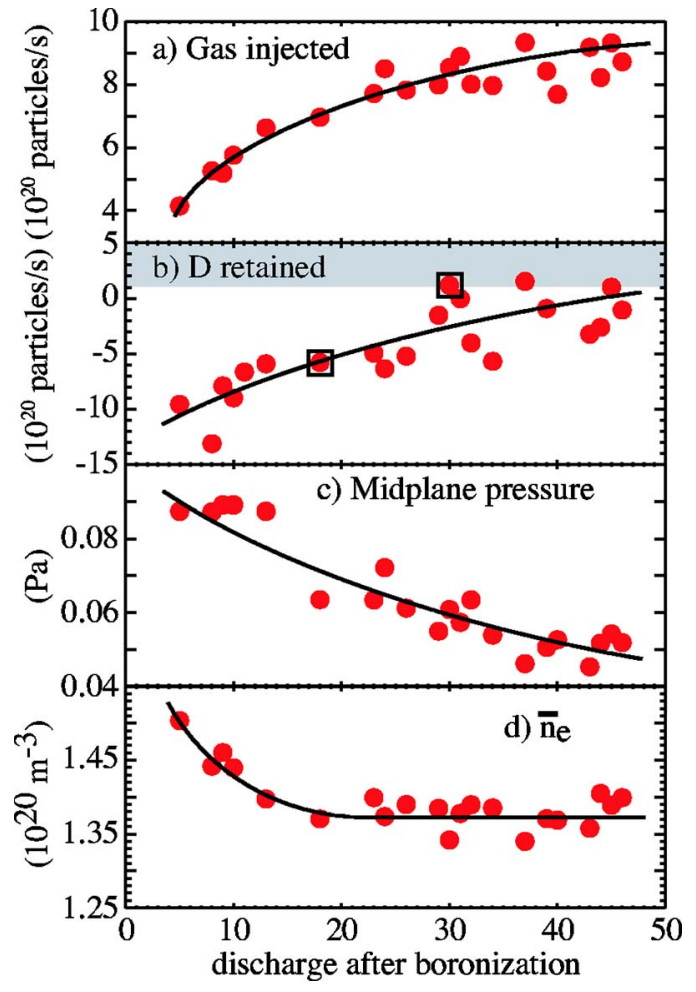

FIG. 10. (Color online) The variation in neutral gas characteristics on a day following overnight boronization vs discharge number. Missing data are either due to problems with the measurement or problems with the discharge (did not breakdown, terminated early, ...). (a) Gas injected; (b) $\mathrm{D}_{2}$ retained. The two data points with open squares are obtained using method 1 as described in the text; (c) midplane pressure; (d) line-averaged density.

$H$-mode density. The gas injected [Fig. 10(a)] to achieve the pre- $H$-mode density increases throughout the day indicating that the boron films were initially fueling the plasma. Boron films from diborane deposition on metals at $T<100{ }^{\circ} \mathrm{C}$ are expected to be saturated with deuterium with $\mathrm{D} / \mathrm{B} \sim 40 \%$. $^{46}$ Thus the films in C-Mod could contain an "additional" D inventory of $\sim 1 \times 10^{23}$ deuterons $/ \mathrm{m}^{2}$ of boron film. (Following boronization with ECDC conditioning in $\mathrm{He}$ of course removes significant amounts of D from the film.) By the end of this set of discharges, the $\mathrm{D}_{2}$ gas injected approaches the level required pre-boronization $(\sim 1$ $\times 10^{21} \mathrm{D}_{2}$ ) for the same discharge characteristics. This indicates that the recycling differences between a boronized and un-boronized Mo vessel are of the order of 50-100 discharges.

There is a concern that the $\mathrm{D}_{2}$ fueling described above might be the result of several hours of ECDC wall conditioning (using $\mathrm{D}_{2}$ ) that is performed after boronization and prior to the above discharge sequence. This seems unlikely based on the experience of several hours of $\mathrm{D}_{2}$ ECDC wall conditioning performed in the morning prior to most run days. In such "standard" discharge conditions the effect on gas fueling is small and lasts primarily for only one discharge, not the many shown in Fig. 10. We believe this is due to the D being deposited in a thin layer at the surface as opposed to boronization where D is likely deposited uniformly throughout the boron layer thickness. The time scales for this reservoir to come to equilibrium with the plasma discharges are 
dependent on the length of discharges, the fluxes and the specifics of D transport within the B layer thickness.

We estimate the amount of $\mathrm{D}_{2}$ retained in the walls after a discharge by two methods: (1) The most accurate method is to close the gate valves to pumps before the discharge, and keep them closed until 5 min after the discharge, when the pressure in the vessel reaches an equilibrium. The amount of gas injected is determined by the pressure drop in calibrated volumes and pressure plena. The difference between the amount injected and the amount remaining is a measure of the injected $\mathrm{D}_{2}$ which is retained in PFC surfaces or fueling; (2) The pumping speed of the torus pumps have been calibrated as a function of the torus pressure. We can then calculate the gas pumped out after a discharge, again comparing with the amount injected to determine the PFC $\mathrm{D}_{2}$ retention/ fueling. Method 2 is less accurate but correctly follows trends. Based on the above methods (method 1 used only for discharges 18 and 30 of Fig. 10) we have determined that PFC surfaces initially fuel the discharge [negative values, Fig. 10(b)], i.e., a recycling coefficient, $R>1$, with the fueling dropping throughout the day.

Variations in the midplane pressure correlate with wall fueling and indicate that the gas is coming from surfaces outside the divertor. Figure 10(c) shows an approximately factor of 2 reduction of the midplane pressure throughout the day occurring over a similar time scale to the variation in gas injected. At the same time the lower divertor pressure only dropped by $\sim 15 \%$. We are not surprised that the divertor pressures are relatively unaffected. Generally it is found that the surfaces outside the divertor in C-Mod play an important role in plasma fueling. ${ }^{47-50}$

The upward trend of Fig. 10(b) continues on following run days (not shown). The level of $\mathrm{D}_{2}$ retention approaches typical values of $3.5-5.0 \times 10^{20} \mathrm{D}_{2}$ per discharge (measured with method 1), i.e., about $50 \%$ of $\mathrm{D}_{2}$ gas fuel injected per discharge. This retention level, like the fuelling requirement, is comparable to that measured pre-boronization. After sufficient plasma operations the boron coating has little or no effect on fuelling and recycling compared to a cleaned high- $Z$ surface. This is akin to the molybdenum impurity behavior, suggesting that relatively small surface areas of the wall are directly affecting the molybdenum source and the recycling.

The similarity of fuel retention between pre- and sufficiently post-boronization discharges is a clue to why the retention is higher than predicted for molybdenum surfaces. The fact that fuel retention does not occur until of order 100 discharges after a boronization is consistent with the time it takes for boron to be eroded from the strike point region of the divertor. ${ }^{32}$ At that point the outer divertor surface material composition could, similar to pre-boronization, to be dominated by molybdenum with similar low levels of boron and other impurities. This provides a plausible reason for similar surface composition pre- and post-boronization. Turning to the actual retention level we must remember that the fuel injection rate is $\sim 100 \times$ lower than the rate of ions impacting divertor surfaces. So, if the gas retention rate is $50 \%$ of that injected, this translates to $\sim 0.5 \%$ of the ion flux to divertor surfaces being retained if the $\mathrm{D}$ retention is peaked there. This is still $20 \times$ higher than the retention rate for $\mathrm{T}$ in tungsten. ${ }^{8,12}$ Obvious differences between a laboratory experiment and the C-Mod experience include the existence of surface temperature transients, impurities on and in the surface, impurities in the plasma, and the variety of ion impact energies that engenders. Since control of hydrogen (tritium) inventory is a major motivation for the use of high-Z metals such as PFCs, this area will be the subject of future investigation.

The characteristics of the SOL are even less affected by boronization than the fueling. SOL profiles of density and temperature were measured throughout a constant discharge day following boronization similar to the run day of Fig. 10. The profiles remained constant from start to end of the run. We interpret this result as meaning that the total gas puff injected from the combination of density feedback and wall fueling stays constant through the day to achieve constant core density. The separatrix density then determines the SOL profiles.

\section{BETWEEN-DISCHARGE BORONIZATION}

Ideally, all PFC surfaces should be well boronized at all times, thus leading to minimal impurities in the core plasma and best plasma performance. The reality is that there are likely small regions, localized both poloidally and toroidally, where the boron erodes quickly. These characteristics point toward the need to understand better where the net erosion of boron and molybdenum is occurring and, if possible, localize the boronization to only those surfaces. Such a localized application of boronization would minimize the buildup of boron in the vessel, thereby minimizing the potential for storing deuterium and unwanted hydrogen. It would also reduce the time needed for boronization and enhance the reproducibility of high-power discharges. Lastly, if we can determine the most important locations for boronization there may be some alternate method for reducing the molybdenum source from such regions, e.g., by sculpting the tile surface to minimize heat loads, by minimizing the sheath rectification of the $\mathrm{rf}$ that leads to high sheath voltages (and sputtering), ${ }^{32}$ by using special materials or coatings or through targeted surface coating/conditioning.

In an effort to accomplish these goals, experiments were carried out to evaluate the efficacy of between-discharge ECDC boronization. All such experiments were scheduled for a day when the effect of the previous overnight boronization was judged to have worn off based on molybdenum radiation and recycling behavior. After an initial background discharge, different length period boronizations were made, each followed by two discharges to evaluate the effectiveness of the boronization and to make sure its effect had worn off before the next between-discharge boronization. The boronization itself was done in a standard fashion, scanning the ECRH resonance from $R=50-103 \mathrm{~cm}$, thus including the outer divertor and regions beyond the ICRF antenna (see Fig. $3)$. Since discharges sometimes had more than one $H$-mode during rf heating (e.g., due to $H$ - $L$ transitions) one measure of success is the neutron production integrated over the period when the rf was on $(0.5 \mathrm{~s})$. There is a clear increase in 

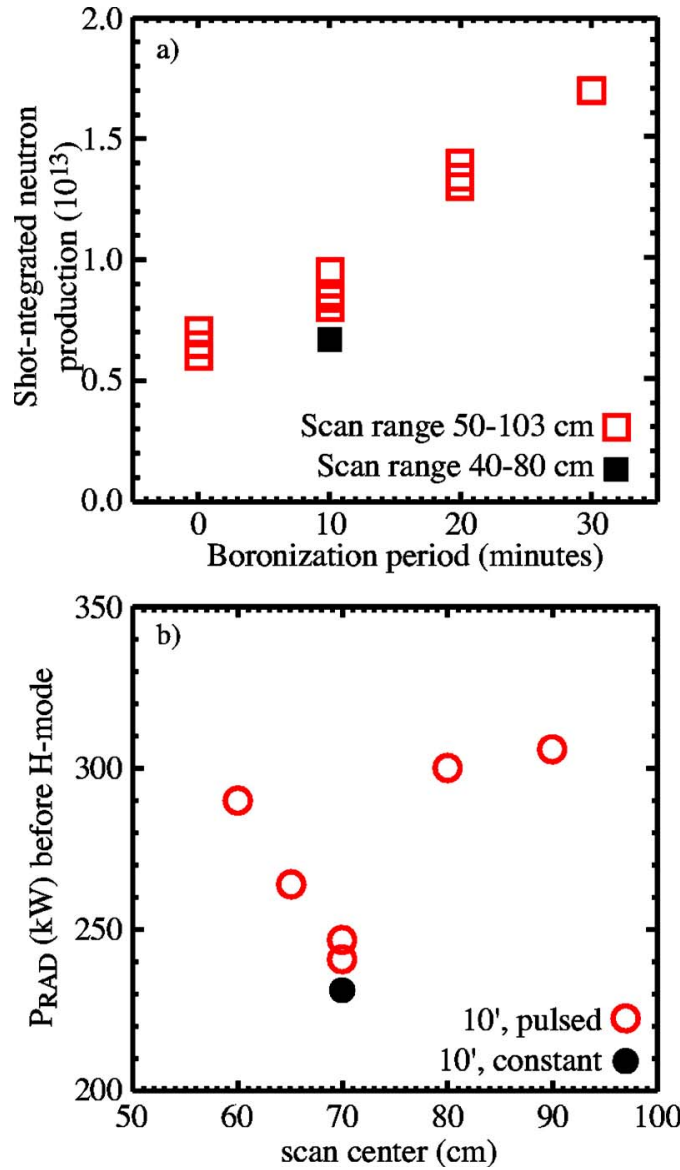

FIG. 11. (Color online) Effect of between-discharge boronization. (a) Variation of total neutrons during the $H$-mode vs boronization period. These boronizations were all done with $50 \%$ duty cycle and utilized a $10 \%$ diborane, $90 \%$ He gas mixture. (b) Variation in radiated power just prior to the $H$-mode transition vs the boronization scan center. These boronization scans were $10 \mathrm{~cm}$ wide (pause at the center of scan) and utilized a 20\% diborane, $80 \% \mathrm{He}$ gas mixture, and a $50 \%$ duty cycle except the one point with $100 \%$ duty cycle.

neutron production with increasing length of boronization [Fig. 11(a)]. All of the second discharges after betweendischarge boronization returned to the un-boronized condition (boronization period of 0 ). That the effect of betweendischarge boronization lasted $\sim 1$ discharge is consistent with overnight boronization. The overnight boronization is performed for a period $\sim 25 \times$ longer than the betweendischarge boronization. The effect of that overnight boronization also lasts $\sim 25 \times$ longer. The times point to a boron erosion rate in the range $5-10 \mathrm{~nm} /$ discharge in the most important areas of localized erosion.

The scan range for one of the $10 \mathrm{~min}$ boronizations cases shown (lowest neutron production) was changed to $R$ $=44-80 \mathrm{~cm}$. The location of the boronization appeared to be important.

The same set of experiments were repeated utilizing between-discharge glow boronization, but with negative results. Possible explanations are that the glow is too diffuse such that the layer is too thin, the glow deposition did not reach important PFC surfaces, or the glow discharge characteristics were not properly optimized.

Having established that the scan range and center may be important, a second set of discharges were then prepared with a narrowed scan width of $10 \mathrm{~cm}$, varying the scan center from boronization to boronization. At the same time the concentration of diborane in the boronization fill gas was increased from $10 \%$ to $20 \%$ ( $\mathrm{He}$ is the remainder of the gas) to increase the boron deposition rate and reduce the boronization period. Figure 11(b) displays the results of this scan where in this case the radiation level during the power ramp just preceding the $H$-mode transition is used as a measure of the boronization effectiveness. The pre- $H$-mode radiation is minimized when the resonance is centered at $70 \mathrm{~cm}$ (while integrated neutrons, or stored energy, would be the inverse of the radiation plot with a peak at $R=70 \mathrm{~cm}$ ). The $R=70 \mathrm{~cm}$ case was visited twice to ensure that the result was reproducible.

A simple interpretation of the data in Fig. 11(b) would indicate that the boronization deposition is peaked at the resonance location rather than matching the density profile measured during deuterium ECDC. If the local deposition rate follows the ECDC density profile-constant for major radii greater than the resonance location, ${ }^{31} R_{\text {Resonance; then }}$ the Fig. 11(b) profile should be a "step" function; low for $R<R_{\text {Resonance }}$ and high for $R>R_{\text {Resonance. }}$. This step function is not observed implying that the boron deposition is not directly tied to the electron density profile and at least somewhat peaked at the resonance location.

The Fig. 11(b) data point to important molybdenum source locations outside the high-heat flux section of the divertor on the low-field side of the plasma. This is consistent with other results mentioned previously: The better correlation of Mo I source rates outside the divertor with core Mo levels; that divertor $\mathrm{N}_{2}$ puffing lowered Mo sources near the outer strike point without affecting core molybdenum levels; the much higher penetration probability of impurities reaching the core plasma when launched from outside the divertor on the outboard side.

Returning to Fig. 3 we see that $R=70 \mathrm{~cm}$ corresponds to the top of the outer divertor as well as the vessel protection tiles at the top of the machine. The poloidal limiters are located at slightly larger major radii (Fig. 3) and cannot be ruled out. Potential processes that could lead to such outboard erosion include plasma fluxes to leading edges, sheath rectification acceleration of ions, and ion orbit losses. Further experiments are needed to clarify the important molybdenum source locations and why. The additional goal is to further optimize between-discharge boronization so as to allow shortened boronization periods.

\section{IMPLICATIONS FOR ITER AND REACTORS}

It is not clear how one might extrapolate these C-Mod results to ITER and beyond. Certainly, the results from a high-Z limiter tokamak are similar to those of C-Mod (Ref. 11) in terms of boronization suppressing Mo levels in the core plasma. However the comparison is not exact because of the complete reliance on limiters as opposed to a divertor, thus placing molybdenum sources with higher penetration probability closer to the plasma. The ASDEX-Upgrade experience is closer to that of C-Mod in terms of having a di- 
vertor; trends of impurity source suppression by boronization are similar. ${ }^{14,51}$ The main difference is that many tungsten surfaces are constantly being coated by carbon eroded from the areas that remain as carbon PFCs. This intrinsic carbon source could lead to the lower level of high-Z impurities in the core plasma that are observed, often a factor of 10-100X lower than Mo in C-Mod. ${ }^{14}$ There are plans to fully-coat all ASDEX-Upgrade PFC surfaces with tungsten. The resulting operational experience should shed some light on whether carbon coating of tungsten is having a major effect.

What are the implications for ITER and reactors? Present methods of boronization are unlikely to be applicable for ITER: long pulses (>500 s) with high energy density will probably wear the coatings off in a period shorter than the ITER pulse. In addition, application of the coating means interrupting the ITER discharge cycle while keeping the toroidal field on. However, ITER, through the selection of Be as the first wall material, has a primarily low-Z PFC wall. Be (and $\mathrm{C}$ from the divertor) will be more easily eroded than $\mathrm{W}$ and, in terms of impurity content, will dominate. This erosion will lead to some amount of low-Z deposition over most PFC surfaces, potentially including those that are originally tungsten. However, if the tungsten-cladded region is a region of strong sputtering and net erosion, then our results suggest that the low- $\mathrm{Z}$ influx will not compete against re-erosion. Based on the JET success with Be gettering, it would certainly seem that the initial ITER mixture of PFC materials (primarily Be) will at least lead to good gettering of oxygen.

Assuming that ITER moves to an all-high-Z PFC machine in support of DEMO, we are less sanguine. The ITER PFC geometry is similar to that of C-Mod both in the divertor shape and the small outboard poloidal limiters. ${ }^{15}$ The ITER SOL opacity to neutrals is also similar to C-Mod (Refs. 19 and 52) implying that impurity penetration could be similar. ITER could have an additional impurity source due to type I ELMs. These are now known to lead to localized PFC-plasma interaction and impurity sources outside the divertor. ${ }^{53-55}$ In summary there are many concerns for all high-Z PFC ITER operation. More operational experience with such reactor-relevant PFC materials will be beneficial to predicting both ITER and commercial reactor operational experience.

\section{DISCUSSION AND SUMMARY}

There are parallels between carbon PFC machines and C-Mod: (1) Boronization replaces one important impurity in the plasma, oxygen in carbon $\mathrm{PFC}$ case and $\mathrm{Mo} / \mathrm{Fe}$ in the C-Mod case, with a low-Z impurity, boron; (2) The reappearance of the underlying PFC impurity species in the plasma is fairly quick - tens of discharges; (3) The majority of PFC and vessel structures remain coated after the first boronization through vacuum breaks and operational campaigns.

The effect of boronization in a high- $Z$ tokamak has some primary differences with its application in carbon PFC machines. Molybdenum is a much more potent core plasma radiator than oxygen (or iron), the impurity most dramatically suppressed in carbon-PFC tokamaks. The reduction of molybdenum in the plasma through boronization leads to large changes in the core impurity levels $\left(n_{\mathrm{Mo}} / n_{e}\right.$ and $n_{\mathrm{Fe}} / n_{e}$ both drop from almost $10^{-3}$ to the mid $10^{-5}$ range), lower plasma radiation and increased energy confinement. The return of core impurity fractions (carbon or molybdenum) to pre-boronization levels thus has much more dramatic effects on radiation in C-Mod due to molybdenum's large atomic number (never fully stripped) and ionization potential which allows radiation losses at much higher $T_{e}$ than the low- $\mathrm{Z}$ impurities. Radiation inside the pedestal reduces the power flow to the edge and, it appears, the edge pedestal and energy confinement.

Boronization has different effects on different impurities and surfaces. A single boronization has longer lasting effects on iron because such surfaces are farther from the plasma and experience lower erosion. Likewise, based on spectroscopic and tile analysis measurements, this is true for the majority of the molybdenum surfaces. An exception is the vertical section of the outer divertor plate where the erosion occurs quickly but does not appear to directly affect the core plasma. In addition it is clear that small areas of molybdenum PFCs, outside the divertor proper, are more rapidly eroded of their boron coating. It is likely that such locations are poloidally and toroidally localized and continually need coating because of the high local erosion rates. Certainly, during vacuum breaks, we have found localized spots on the poloidal limiters and antenna protection limiters that are melted or devoid of boron.

Between-discharge ECDC boronization may be a technique for more directly determining the important erosion locations and replace the rapidly eroding boron. Initial experiments with this technique have been successful in replacing the eroded boron where needed. More experiments are needed to better localize the effect and reduce the time needed between discharges.

Boronization has short-term effects on recycling as well as on core impurity levels. The boronization loads the new boron layer with $\mathrm{D}$ which, over a series of $50-100$ discharges, is reduced back to the "normal" level as it fuels the plasma. Long after the boronization, the PFC surfaces retain D during a discharge; up to $50 \%$ of that injected. This level of $\mathrm{D}$ retention is similar to uncoated surfaces. Over the course of a day or days the amount retained is much lower due to random disruptions that normally occur. Since the retention process is not well understood we cannot yet extrapolate to operation with high-Z PFCs in ITER or a reactor.

While operation with un-boronized high-Z PFCs led to high impurity levels and poor energy confinement in the C-Mod, a better understanding of the erosion processes is needed to extrapolate to future experiments. Although the applicability of current boronization techniques to ITER is in doubt it may be possible to develop some localized version that could be useful for such a machine.

\section{ACKNOWLEDGMENTS}

Alcator C-Mod's contributions to fusion energy science are made possible by the excellent engineers, technical staff, students, and scientists on the Alcator team. The authors would also like to thank Arne Kallenbach and Ralph Dux 
who supplied atomic rate data and with whom we had several useful discussions. Discussions with Russ Doerner and Graham Wright on the subject of D retention were especially helpful.

This work is supported by the U.S. Department of Energy Cooperative Agreement No. DE-FC02-99ER54512.

${ }^{1}$ F. W. Perkins, D. E. Post, N. A. Uckan et al., Nucl. Fusion 39, 2137 (1999).

${ }^{2}$ C. H. Wu, J. P. Bonal, H. Kwast et al., Fusion Eng. Des. 39, 263 (1998).

${ }^{3}$ V. Barabash, G. Federici, M. Rödig et al., J. Nucl. Mater. 283, 138 (2000).

${ }^{4}$ G. Federici, J. N. Brooks, D. P. Coster et al., J. Nucl. Mater. 290, 260 (2001).

${ }^{5}$ H. Bolt, V. Barabash, G. Federici et al., J. Nucl. Mater. 307, 43 (2002).

${ }^{6}$ V. Barabash, G. Federici, J. Linke et al., J. Nucl. Mater. 313, 42 (2003).

${ }^{7}$ M. Gasparotto, R. Andreani, L. V. Boccaccini et al., Fusion Eng. Des. 66, 129 (2003).

${ }^{8}$ R. Causey, K. Wilson, T. Venhaus et al., J. Nucl. Mater. 266, 467 (1999).

${ }^{9}$ S. Itoh, K. N. Sato, K. Nakamura et al., Nucl. Fusion 39, 1257 (1999).

${ }^{10}$ M. Sakamoto, S. Itoh, K. Nakamura et al., Nucl. Fusion 42, 165 (2002).

${ }^{11}$ M. L. Apicella, G. Mazzitelli, B. Esposito et al., Nucl. Fusion 45, 685 (2005).

${ }^{12}$ R. A. Causey, C. L. Kunz, and D. F. Cowgill, J. Nucl. Mater. 337, 600 (2005).

${ }^{13}$ V. Philipps, A. Pospieszczyk, M. Z. Tokar et al., in Proceedings of the 15th IAEA Conference on Plasma Physics and Controlled Fusion Research, Seville, Spain, 1994 (IAEA, Vienna, 1995), Vol. 2, pp. 149-157.

${ }^{14}$ R. Neu, R. Dux, A. Geier et al., Plasma Phys. Controlled Fusion 44, 811 (2002).

${ }^{15}$ ITER Physics Expert group on divertor, ITER Physics Expert group on divertor modelling and database, and ITER Physics Basis Editors, Nucl. Fusion 39, 2391 (1999).

${ }^{16}$ J. Winter, Plasma Phys. Controlled Fusion 38, 1503 (1996).

${ }^{17}$ K. J. Dietz, Plasma Phys. Controlled Fusion 32, 837 (1990).

${ }^{18}$ M. Keilhacker, Phys. Fluids B 2, 1291 (1990).

${ }^{19}$ A. S. Kukushkin, H. D. Pacher, G. W. Pacher et al., Nucl. Fusion 43, 716 (2003).

${ }^{20}$ Y. Lin, see EPAPS Document No. E-PHPAEN-13-056691. This document can be reached via a direct link in the online article's HTML reference section or via the EPAPS homepage (http://www.aip.org/ pubservs.epaps.html).

${ }^{21}$ I. H. Hutchinson, R. Boivin, F. Bombarda et al., Phys. Plasmas 1, 1511 (1994).

${ }^{22}$ S. N. Golovato, M. Porkolab, Y. Takase et al., in Proceedings of the 11th Topical Conference on Radio Frequency in Plasmas, Palm Springs, CA, 1996 (American Institute of Physics), Vol. 355, pp. 23-30.

${ }^{23}$ S. J. Wukitch, R. L. Boivin, P. T. Bonoli et al., Plasma Phys. Controlled Fusion 46, 1479 (2004).

${ }^{24}$ J. A. Goetz, B. Lipschultz, M. A. Graf et al., J. Nucl. Mater. 220, 971 (1995).

${ }^{25}$ R. L. Boivin, J. A. Goetz, E. S. Marmar et al., Rev. Sci. Instrum. 70, 260 (1999).

${ }^{26}$ Russell A. Hulse, Nucl. Technol./Fusion 3, 259 (1983).

${ }^{27}$ J. E. Rice, J. L. Terry, J. A. Goetz et al., Phys. Plasmas 4, 1605 (1997).
${ }^{28}$ D. E. Post, R. V. Jensen, C. B. Tarter et al., At. Data Nucl. Data Tables 20, 397 (1977)

${ }^{29}$ K. B. Fournier, D. Pacella, M. J. May et al., Nucl. Fusion 37, 825 (1997).

${ }^{30}$ K. B. Fournier, D. Pacella, M. May et al., Nucl. Fusion 38, 639 (1998).

${ }^{31}$ R. T. Nachtrieb, B. L. LaBombard, J. L. Terry et al., J. Nucl. Mater. 266, 896 (1999).

${ }^{32}$ B. Lipschultz, D. A. Pappas, B. LaBombard et al., Nucl. Fusion 41, 585 (2001).

${ }^{33}$ W. R. Wampler, B. LaBombard, B. Lipschultz et al., J. Nucl. Mater. 266, 217 (1999).

${ }^{34}$ T. Tanabe, Y. Furuyama, N. Saitoh et al., Trans. Jpn. Inst. Met. 28, 706 (1987).

${ }^{35}$ T. Noda, H. Araki, and H. Suzuki, Fusion Sci. Technol. 41, 962 (2002).

${ }^{36}$ P. N. Yushmanov, T. Takizuka, K. S. Riedel et al., Nucl. Fusion 30, 1999 (1990).

${ }^{37}$ D. K. Mansfield, K. W. Hill, J. D. Strachan et al., Phys. Plasmas 3, 1892 (1996).

${ }^{38}$ M. Greenwald, R. L. Boivin, F. Bombarda et al., Nucl. Fusion 37, 793 (1997).

${ }^{39}$ C. S. Pitcher, A. H. Boozer, H. Murmann et al., Phys. Plasmas 4, 2577 (1997).

${ }^{40}$ T. H. Osborne, R. J. Groebner, L. L. Lao et al., Plasma Phys. Controlled Fusion 40, 845 (1998).

${ }^{41}$ A. E. Hubbard, B. Lipschultz, D. Mossessian et al., in Proceedings of the 26th EPS Conference on Controlled Fusion and Plasma Physics, Maastricht, Netherlands, 1999 (European Physical Society, Geneva, 1999), Vol. 23J, pp. 13-16.

${ }^{42}$ H. P. Summer, "Atomic Data and Analysis Structure User Manual," JET Joint Undertaking Report No. IR(94)96 (1994).

${ }^{43}$ F. Waelbroeck, T. Banno, H. G. Esser et al., J. Nucl. Mater. 162, 496 (1989).

${ }^{44}$ F. Waelbroeck, J. Winter, G. Esser et al., Plasma Phys. Controlled Fusion 31, 185 (1989).

${ }^{45}$ J. Winter, H. G. Esser, L. Konen et al., J. Nucl. Mater. 162, 713 (1989).

${ }^{46}$ K. Tsuzuki, M. Natsir, N. Inoue et al., J. Nucl. Mater. 241, 1055 (1997).

${ }^{47}$ B. Labombard, B. Lipschultz, J. Goetz et al., in Proceedings of the 18th International Conference on Plasma Physics and Controlled Fusion Research, IAEA, Sorrento, Italy, 2000 (IAEA, Vienna, 2001), Vol. 1, p. EX5/6.

${ }^{48}$ B. LaBombard, M. V. Umansky, R. L. Boivin et al., Nucl. Fusion 40, 2041 (2000).

${ }^{49}$ B. Lipschultz, B. LaBombard, C. S. Pitcher et al., Plasma Phys. Controlled Fusion 44, 733 (2002).

${ }^{50}$ B. Lipschultz, D. Whyte, and B. LaBombard, Plasma Phys. Controlled Fusion 47, 1559 (2005).

${ }^{51}$ A. Kallenbach, R. Neu, R. Dux et al., Plasma Phys. Controlled Fusion 47, B207 (2005).

${ }^{52}$ B. Lipschultz, P. Andrew, J. Coad et al., in Proceedings of the 30th European Conference on Controlled Fusion and Plasma Physics, St. Petersburg, Russia, 2003 (European Physical Society, Geneva, 2003), Vol. 27A, p. 3.197.

${ }^{53}$ A. Herrmann, T. Eich, V. Rohde et al., Plasma Phys. Controlled Fusion 46, 971 (2004).

${ }^{54}$ A. Herrmann, J. Neuhauser, V. Rohde et al., J. Nucl. Mater. 337-339, 697 (2005).

${ }^{55}$ A. Kirk, H. R. Wilson, R. Akers et al., Plasma Phys. Controlled Fusion 47, 315 (2005). 